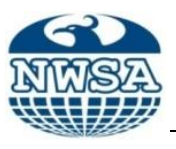

NWSA-Physical Sciences

ISSN : $1306-3111 / 1308-7304$

NWSA ID: 2015.10.1.3A0067

Status : Original Study

Received: October 2014

E-Journal of New World Sciences Academy

Mehdi Jafari

University College of Science and Technology Elm o Fan, Urmia-Iran mj_msc@yahoo.com

http://dx.doi.org/10.12739/NWSA.2015.10.1.3A0067

\title{
MATRIX ALGEBRAS IN $\mathrm{E}_{\alpha \beta}^{4}$ AND THEIR APPLICATIONS
}

\section{ABSTRACT}

By Hamilton operators, generalized quaternions have been expressed in terms of $4 \times 4$ matrices. In this paper, geometric applications of these matrices in generalized 4-space $\mathrm{E}_{\alpha \beta}^{4}$ are given. We also show that the set of these matrices with the group operation of matrix multiplication is Lie group of 6-dimension.

Keywords: De Moivre's Formula, Homothetic Motion, Lie Group, Rotation, Matrix

\section{$\mathbf{E}_{\alpha \beta}^{4}{ }^{\prime}$ DE MATRIS CEBİİ VE UYGULAMALARI}

ÖZET

Hamilton operatorleri ile bir gelişmiş kuaterniyon $4 \times 4$ matrisleri ile gösterilmiştir. Bu makalede matrislerin uygulamaları gelişmiş uzay'da verilmiştir. Ayrıca, bu matrislerin kümesi matris çarpım ile altı boyutlu bir Lie grubu oluşturulmuştur.

Anahtar Kelimeler: De Moivre's Formülü, Homothetik Hareket, Lie Grubu, Dönme, Matris 


\begin{abstract}
1. INTRRODUCTION (GIRİ̧)
The quaternions are commonly used in physics, chemistry, robotics, mechanics and electronics. A brief introduction of the generalized quaternions is provided in [5], the subject which have investigated in algebra [6]. The generalized quaternion algebra is an associative and non-commutative 4-dimensional clifford algebra. Recently, we have studied the generalized quaternion and some of their algebraic properties [1]. A matrix corresponding to Hamilton operators, defined for the generalized quaternions, determines a Homothetic motion and also can be used to described the rotation in 4dimensional space in $E_{\alpha \beta}^{4}$. In addition, by De-Moivre's formula every power of this matrix is immediately obtained.
\end{abstract}

\title{
2. RESEARCH SIGNIFICANCE (ÇALIŞMANIN ÖNEMI)
}

In this work, after a review of some fundamental properties of the generalized quaternions, we study the applications of matrices corresponding to generalized quaternions. The set of these matrices with the group operation of matrix multiplication is Lie group of 6dimension. Finally, we give some example for the purpose of more clarification.

\section{EXPERIMENTAL METHOD-PROCESS (DENEYSEL ÇALIŞMA)}

In this section, we define a new inner product and give a brief summary of the generalized quaternions.

- Definition (Tanım) 1: Let $\vec{u}=\left(u_{1}, u_{2}, u_{3}, u_{4}\right), \vec{v}=\left(v_{1}, v_{2}, v_{3}, v_{4}\right) \in \mathrm{R}^{4}$. If $\quad \alpha, \beta \in \mathrm{R}^{+}$, the generalized inner product of $\vec{u}$ and $\vec{v}$ is defined by

$$
\langle\vec{u}, \vec{v}\rangle=u_{1} v_{1}+\alpha u_{2} v_{2}+\beta u_{3} v_{3}+\alpha \beta u_{4} v_{4} .
$$

It could be written

$$
\langle\vec{u}, \vec{v}\rangle=u^{T}\left[\begin{array}{cccc}
1 & 0 & 0 & 0 \\
0 & \alpha & 0 & 0 \\
0 & 0 & \beta & 0 \\
0 & 0 & 0 & \alpha \beta
\end{array}\right]=u^{T} G v
$$

Also, if $\alpha>0, \beta<0,\langle\vec{u}, \vec{v}\rangle$ is called the generalized Lorentz an inner product. The vector space on $\mathrm{R}^{4}$ equipped with the generalized inner product is called 4-dimensional generalized space and denoted by $\mathrm{E}_{\alpha \beta}^{4}$.

- Definition (Tanım) 2: A matrix $A$ is called a quasi-orthogonal matrix if $A^{T} \varepsilon A=\varepsilon$ and $\operatorname{det} A=1$ where

$$
\varepsilon=\left[\begin{array}{cccc}
1 & 0 & 0 & 0 \\
0 & \alpha & 0 & 0 \\
0 & 0 & \beta & 0 \\
0 & 0 & 0 & \alpha \beta
\end{array}\right],
$$

And $\alpha, \beta \in \mathrm{R}$. the set of all quasi-orthogonal matrices, $\mathrm{QO}(3)$, with the operation of matrix multiplication is called rotations group in 4spaces $\mathrm{E}_{\alpha \beta}^{4}[2]$. 


\section{Jafari, $M$. NWSA-Physical Sciences, 3A0067, 10, (1), 1-13.}

- Definition (Tanım) 3: A generalized quaternion $q$ is an expression of the form

$$
q=a_{0}+a_{1} \vec{i}+a_{2} \vec{j}+a_{3} \vec{k}
$$

Where $a_{0}, a_{1}, a_{2}$ and $a_{3}$ are real numbers and $\vec{i}, \vec{j}, \vec{k}$ are quaternionic units satisfying the equalities

and

$$
\begin{aligned}
& \vec{i}^{2}=-\alpha, \quad \vec{j}^{2}=-\beta, \quad \vec{k}^{2}=-\alpha \beta, \\
& \overrightarrow{i j}=\vec{k}=-\overrightarrow{j i}, \quad \vec{j} \vec{k}=\beta \vec{i}=-\overrightarrow{k j},
\end{aligned}
$$

$$
\vec{k} \vec{i}=\alpha \vec{j}=-\overrightarrow{i k}, \quad \alpha, \beta \in \mathrm{R} .
$$

The set of all generalized quaternions is denoted by $\mathrm{H}_{\alpha \beta}$. We express the basic operations in the $\vec{i}, \vec{j}, \vec{k}$ form. The addition becomes as

$$
\begin{aligned}
\left(a_{0}+a_{1} \vec{i}+a_{2} \vec{j}+a_{3} \vec{k}\right) & +\left(b_{0}+b_{1} \vec{i}+b_{2} \vec{j}+b_{3} \vec{k}\right) \\
= & \left(a_{0}+b_{0}\right)+\left(a_{1}+b_{1}\right) \vec{i}+\left(a_{2}+b_{2}\right) \vec{j}+\left(a_{3}+b_{3}\right) \vec{k}
\end{aligned}
$$

and the multiplication as

$$
\begin{aligned}
\left(a_{0}+a_{1} \vec{i}+a_{2} \vec{j}+a_{3} \vec{k}\right) & \left(b_{0}+b_{1} \vec{i}+b_{2} \vec{j}+b_{3} \vec{k}\right) \\
& =\left(a_{0} b_{0}-\alpha a_{1} b_{1}-\beta a_{2} b_{2}-\alpha \beta a_{3} b_{3}\right) \\
& +\left(a_{1} b_{0}+a_{0} b_{1}-\beta a_{3} b_{2}+\beta a_{2} b_{3}\right) \vec{i} \\
& +\left(a_{2} b_{0}+\alpha a_{3} b_{1}+a_{0} b_{2}-\alpha a_{1} b_{3}\right) \vec{j} \\
& +\left(a_{3} b_{0}-a_{2} b_{1}+a_{1} b_{2}+a_{0} b_{3}\right) \vec{k}
\end{aligned}
$$

Given $q=a_{0}+a_{1} \vec{i}+a_{2} \vec{j}+a_{3} \vec{k}$, $a_{0}$ is called the scalar part of $q$, denoted by $S(q)=a_{0}$

and $a_{1} \vec{i}+a_{2} \vec{j}+a_{3} \vec{k}$ is called the vector part of $q$, denoted by

The conjugate of $q$ is

$$
\vec{V}(q)=a_{1} \vec{i}+a_{2} \vec{j}+a_{3} \vec{k}
$$

$$
\bar{q}=a_{0}-a_{1} \vec{i}-a_{2} \vec{j}-a_{3} \vec{k}
$$

The norm of $q$ is

$$
N_{q}=\bar{q} q=q \bar{q}=a_{0}^{2}+\alpha a_{1}^{2}+\beta a_{2}^{2}+\alpha \beta a_{3}^{2} .
$$

The inverse of $q$ with $N_{q} \neq 0$, is

$$
q^{-1}=\frac{1}{N_{q}} \bar{q} .
$$

Clearly $q q^{-1}=1+0 \vec{i}+0 \vec{j}+0 \vec{k}$. Note also that $\overline{q p}=\bar{p} \bar{q}$ and $(q p)^{-1}=p^{-1} q^{-1}$ [1]

- Definition (Tanım) 4: A Lie group is a group G, equipped with a manifold structure such that the group operations

Mult: $G \times G \rightarrow G, \quad\left(g_{1}, g_{2}\right) \rightarrow g_{1} g_{2}$

Inv: $\mathrm{G} \rightarrow \mathrm{G}, \mathrm{g} \rightarrow \mathrm{g}^{-1}$ are smooth. 
For example, the general linear group

$$
\mathrm{GL}(n, \square)=\left\{A \in \operatorname{Mat}_{n}(\mathrm{R}): \operatorname{det} A \neq 0\right\}
$$

is an open subset of $\operatorname{Mat}_{n}(\mathrm{R})$, hence a sub manifold, and the smoothness of group multiplication follows since the product map for $\operatorname{Mat}_{n}(\mathrm{R})$, is obviously smooth[4].

- Definition (Tanım) 5:Left multiplication by a generalized quaternion $q$ is a linear map

$$
\stackrel{+}{h}_{q}(x)=q x, \quad x \in \mathrm{H}_{\alpha \beta},
$$

from the quaternions into the quaternions, as is right multiplication,

$$
\begin{aligned}
& \qquad \bar{h}_{q}(x)=x q \quad x \in \mathrm{H}_{\alpha \beta} . \\
& \text { Since these multiplications are linear maps from four } \\
& \text { dimensional vector space into itself, we can find a matrix } \\
& \text { representation of each. } \\
& \text { The Hamilton operators } \stackrel{+}{H} \text { and } \bar{H} \text {, could be represented as the matrices; }
\end{aligned}
$$

and

$$
\stackrel{+}{H}(q)=\left[\begin{array}{cccc}
a_{0} & -\alpha a_{1} & -\beta a_{2} & -\alpha \beta a_{3} \\
a_{1} & a_{0} & -\beta a_{3} & \beta a_{2} \\
a_{2} & \alpha a_{3} & a_{0} & -\alpha a_{1} \\
a_{3} & -a_{2} & a_{1} & a_{0}
\end{array}\right]
$$

$$
\bar{H}(q)=\left[\begin{array}{cccc}
a_{0} & -\alpha a_{1} & -\beta a_{2} & -\alpha \beta a_{3} \\
a_{1} & a_{0} & \beta a_{3} & -\beta a_{2} \\
a_{2} & -\alpha a_{3} & a_{0} & \alpha a_{1} \\
a_{3} & a_{2} & -a_{1} & a_{0}
\end{array}\right] .
$$

- Theorem (Teorem) 1: If $q$ and $p$ are two real quaternions, $\lambda$ is a real number and $\stackrel{+}{H}$ and $\bar{H}$ are operators as defined in equations (1) and (2), respectively, then the following identities hold:

1. $q=p \Leftrightarrow \stackrel{+}{H}(q)=\stackrel{+}{H}(p) \Leftrightarrow \bar{H}(q)=\bar{H}(p)$.

2. $\stackrel{+}{H}(q+p)=\stackrel{+}{H}(q)+\stackrel{+}{H}(p), \quad \bar{H}(q+p)=\bar{H}(q)+\bar{H}(p)$.

3. $\stackrel{+}{H}(\lambda q)=\lambda \stackrel{+}{H}(q), \bar{H}(\lambda q)=\lambda \bar{H}(q)$.

4. $\stackrel{+}{H}(q p)=\stackrel{+}{H}(q) \stackrel{+}{H}(p), \quad \bar{H}(q p)=\bar{H}(p) \bar{H}(q)$.

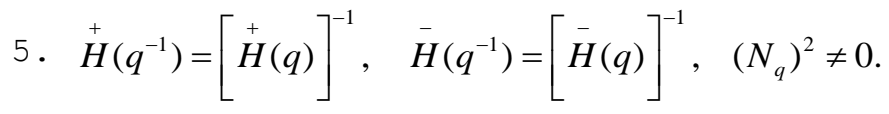

6. $\stackrel{+}{H}(\bar{q})=[\stackrel{+}{H}(q)]^{T}, \quad \bar{H}(\bar{q})=[\bar{H}(q)]^{T}$. 
7. $\operatorname{det}[\stackrel{+}{H}(q)]=\left(N_{q}\right)^{2}, \operatorname{det}[\stackrel{-}{H}(q)]=\left(N_{q}\right)^{2}$.

8. $\operatorname{tr}[\stackrel{+}{H}(q)]=4 a_{0}, \quad \operatorname{tr}[\stackrel{-}{H}(q)]=4 a_{0}$.

Proof: The proof can be found in [1].

- Theorem (Teorem) 2: The map

defined as

$$
\psi:\left(\mathrm{H}_{\alpha \beta},+, .\right) \rightarrow\left(\mathrm{M}_{(4, \mathrm{R})}, \oplus, \otimes\right)
$$

is an isomorphism of algebras.

$$
\psi\left(a_{0}+a_{1} \overrightarrow{\mathrm{i}}+a_{2} \overrightarrow{\mathrm{j}}+a_{3} \overrightarrow{\mathrm{k}}\right) \mapsto\left[\begin{array}{cccc}
a_{0} & -\alpha a_{1} & -\beta a_{2} & -\alpha \beta a_{3} \\
a_{1} & a_{0} & -\beta a_{3} & \beta a_{2} \\
a_{2} & \alpha a_{3} & a_{0} & -\alpha a_{1} \\
a_{3} & -a_{2} & a_{1} & a_{0}
\end{array}\right]
$$

Proof: See [7] for a similar proof.

- Theorem (Teorem) 3: Let

$$
\Omega=\left\{A=\left[\begin{array}{cccc}
x_{0} & -\alpha x_{1} & -\beta x_{2} & -\alpha \beta x_{3} \\
x_{1} & x_{0} & -\beta x_{3} & \beta x_{2} \\
x_{2} & \alpha x_{3} & x_{0} & -\alpha x_{1} \\
x_{3} & -x_{2} & x_{1} & x_{0}
\end{array}\right]: x_{i} \in \square, 1 \leq i \leq 4\right\} .
$$

Then $\Omega$ is a differentiable manifold.

Proof: Let us consider the following function:

$$
\begin{aligned}
f: \Omega & \rightarrow \mathrm{E}_{\alpha \beta}^{4} \\
A & \rightarrow f(A)=\left(x_{0}, x_{1}, x_{2}, x_{3}\right),
\end{aligned}
$$

$f$ is one-to-one and on to function, and since $f(\Omega)=\mathrm{E}_{\alpha \beta}^{4}$ then $f(\Omega)$ is open set. Furthermore, since $x_{i}, i=1,2,3,4$ are continuously, and then $f, f^{-1}$ are continuously functions. $\{(f, \Omega)\}$ is a differentiable atlas with one chart, so $\Omega$ is a differentiable manifold.

- Theorem (Teorem) 4: Under matrix multiplication, $\Omega^{*}=\Omega-\{0\}$ is a Lie group of dimension 6 .

Proof: $\Omega^{*}$ under matrix multiplication is a matrix group. Also, $\Omega^{*}$ is a sub manifold of $\Omega$. Furthermore, the group operations

are obviously smooth.

$$
\begin{aligned}
\text { Mult }: & \Omega^{*} \times \Omega^{*} \rightarrow \Omega^{*}, \quad\left(A_{1}, A_{2}\right) \rightarrow A_{1} A_{2} \\
& \text { Inv: } \Omega^{*} \rightarrow \Omega^{*}, \quad A \rightarrow A^{-1}
\end{aligned}
$$


Let us find the left algebra, i.e., the tangent space at the unit elementary, $\mathrm{T}_{e}\left(\Omega^{*}\right)$. Let us consider the map $\Phi: \mathrm{E}_{\alpha \beta}^{4}-\{0\} \rightarrow \Omega^{*}$, defined by

$$
\Phi\left(x_{1}, x_{2}, x_{3}, x_{4}\right)=\left[\begin{array}{cccc}
x_{0} & -\alpha x_{1} & -\beta x_{2} & -\alpha \beta x_{3} \\
x_{1} & x_{0} & -\beta x_{3} & \beta x_{2} \\
x_{2} & \alpha x_{3} & x_{0} & -\alpha x_{1} \\
x_{3} & -x_{2} & x_{1} & x_{0}
\end{array}\right] .
$$

For point $p=(1,0,0,0), \Phi(p)=e=I_{4}$ is identity element of $\Omega^{*}$.

- Theorem (Teorem) 5: Consider the map $\left.\Phi_{*}\right|_{P}: \mathrm{T}_{\mathrm{p}} \mathrm{E}_{\alpha \beta}^{4} \rightarrow \mathrm{T}_{\Phi(\mathrm{p})} \Omega^{*}$. This map is one-to-one.

Proof: If we show that $\Phi_{*}\left(V_{p}\right)=0 \Rightarrow V_{p}=0$ then Theorem is proved. For every $V_{p} \in \mathrm{T}_{\mathrm{p}} \mathrm{E}_{\alpha \beta}^{4}$, we have $V_{p}=a_{1} \partial / \partial x_{1}+a_{2} \partial / \partial x_{2}+a_{3} \partial / \partial x_{3}+a_{4} \partial / \partial x_{4}$, so

$$
\begin{aligned}
\left.\Phi_{*}\right|_{P}\left(\mathrm{~V}_{\mathrm{p}}\right) & =\left[\begin{array}{cccc}
\mathrm{V}_{\mathrm{p}}\left[\mathrm{x}_{1}\right] & \mathrm{V}_{\mathrm{p}}\left[-\alpha \mathrm{x}_{2}\right] & \mathrm{V}_{\mathrm{p}}\left[-\beta \mathrm{x}_{3}\right] & \mathrm{V}_{\mathrm{p}}\left[-\alpha \beta \mathrm{x}_{4}\right] \\
\mathrm{V}_{\mathrm{p}}\left[\mathrm{x}_{2}\right] & \mathrm{V}_{\mathrm{p}}\left[\mathrm{x}_{1}\right] & \mathrm{V}_{\mathrm{p}}\left[-\beta \mathrm{x}_{4}\right] & \mathrm{V}_{\mathrm{p}}\left[\beta \mathrm{x}_{3}\right] \\
\mathrm{V}_{\mathrm{p}}\left[\mathrm{x}_{3}\right] & \mathrm{V}_{\mathrm{p}}\left[\alpha \mathrm{x}_{4}\right] & \mathrm{V}_{\mathrm{p}}\left[\mathrm{x}_{1}\right] & \mathrm{V}_{\mathrm{p}}\left[-\alpha \mathrm{x}_{2}\right] \\
\mathrm{V}_{\mathrm{p}}\left[\mathrm{x}_{4}\right] & \mathrm{V}_{\mathrm{p}}\left[-\mathrm{x}_{3}\right] & \mathrm{V}_{\mathrm{p}}\left[\mathrm{x}_{2}\right] & \mathrm{V}_{\mathrm{p}}\left[\mathrm{x}_{1}\right]
\end{array}\right] \\
& =\left[\begin{array}{cccc}
a_{1} & -\alpha a_{2} & -\beta a_{3} & -\alpha \beta a_{4} \\
a_{2} & a_{1} & -\beta a_{4} & \beta a_{3} \\
a_{3} & \alpha a_{4} & a_{1} & -\alpha a_{2} \\
a_{4} & -a_{3} & a_{2} & a_{1}
\end{array}\right]=[0]
\end{aligned}
$$

Then $a_{1}=a_{2}=a_{3}=a_{4}=0$. So, $\Phi_{*}$ an injective map. On the other hand, $\operatorname{dimT}_{\mathrm{p}} \mathrm{E}_{\alpha \beta}^{4}=\operatorname{dimT}_{e} \Omega^{*}=4$, thus, $\Phi_{*}$ is a linear isomorphism. Since every linear isomorphism maps any basis of space to another one. So we determine the basis of space $\mathrm{T}_{\Phi(\mathrm{p})} \Omega^{*}$.

It is obviously that $\mathrm{T}_{\mathrm{p}} \mathrm{E}_{\alpha \beta}^{4}=\operatorname{Sp}\left\{\partial / \partial x_{1}, \partial / \partial x_{2}, \partial / \partial x_{3}, \partial / \partial x_{4}\right\}$. We find the image of this basis under the map $\Phi_{*}$.

$$
\begin{gathered}
\left.\Phi_{*}\right|_{P}\left(\frac{\partial}{\partial x_{1}}\right)=\frac{\partial}{\partial x_{1}}\left[\begin{array}{cccc}
x_{1} & -\alpha x_{2} & -\beta x_{3} & -\alpha \beta x_{4} \\
x_{2} & x_{1} & -\beta x_{4} & \beta x_{3} \\
x_{3} & \alpha x_{4} & x_{1} & -\alpha x_{2} \\
x_{4} & -x_{3} & x_{2} & x_{1}
\end{array}\right]=\left[\begin{array}{cccc}
1 & 0 & 0 & 0 \\
0 & 1 & 0 & 0 \\
0 & 0 & 1 & 0 \\
0 & 0 & 0 & 1
\end{array}\right] \\
\left.\Phi_{*}\right|_{P}\left(\frac{\partial}{\partial x_{2}}\right)=\frac{\partial}{\partial x_{2}}\left[\begin{array}{cccc}
x_{1} & -\alpha x_{2} & -\beta x_{3} & -\alpha \beta x_{4} \\
x_{2} & x_{1} & -\beta x_{4} & \beta x_{3} \\
x_{3} & \alpha x_{4} & x_{1} & -\alpha x_{2} \\
x_{4} & -x_{3} & x_{2} & x_{1}
\end{array}\right]=\left[\begin{array}{cccc}
0 & -\alpha & 0 & 0 \\
1 & 0 & 0 & 0 \\
0 & 0 & 0 & -\alpha \\
0 & 0 & 1 & 0
\end{array}\right]
\end{gathered}
$$




$$
\begin{gathered}
\left.\Phi_{*}\right|_{P}\left(\frac{\partial}{\partial x_{3}}\right)=\frac{\partial}{\partial x_{3}}\left[\begin{array}{cccc}
x_{1} & -\alpha x_{2} & -\beta x_{3} & -\alpha \beta x_{4} \\
x_{2} & x_{1} & -\beta x_{4} & \beta x_{3} \\
x_{3} & \alpha x_{4} & x_{1} & -\alpha x_{2} \\
x_{4} & -x_{3} & x_{2} & x_{1}
\end{array}\right]=\left[\begin{array}{cccc}
0 & 0 & -\beta & 0 \\
0 & 0 & 0 & \beta \\
1 & 0 & 0 & 0 \\
0 & -1 & 0 & 0
\end{array}\right] \\
\left.\Phi_{*}\right|_{P}\left(\frac{\partial}{\partial x_{4}}\right)=\frac{\partial}{\partial x_{4}}\left[\begin{array}{cccc}
x_{1} & -\alpha x_{2} & -\beta x_{3} & -\alpha \beta x_{4} \\
x_{2} & x_{1} & -\beta x_{4} & \beta x_{3} \\
x_{3} & \alpha x_{4} & x_{1} & -\alpha x_{2} \\
x_{4} & -x_{3} & x_{2} & x_{1}
\end{array}\right]=\left[\begin{array}{cccc}
0 & 0 & 0 & -\alpha \beta \\
0 & 0 & -\beta & 0 \\
0 & \alpha & 0 & 0 \\
1 & 0 & 0 & 0
\end{array}\right]
\end{gathered}
$$

So we have

$$
\mathrm{T}_{e} \Omega^{*}=\operatorname{Sp}\left\{\left[\begin{array}{cccc}
1 & 0 & 0 & 0 \\
0 & 1 & 0 & 0 \\
0 & 0 & 1 & 0 \\
0 & 0 & 0 & 1
\end{array}\right],\left[\begin{array}{cccc}
0 & -\alpha & 0 & 0 \\
1 & 0 & 0 & 0 \\
0 & 0 & 0 & -\alpha \\
0 & 0 & 1 & 0
\end{array}\right],\left[\begin{array}{cccc}
0 & 0 & -\beta & 0 \\
0 & 0 & 0 & \beta \\
1 & 0 & 0 & 0 \\
0 & -1 & 0 & 0
\end{array}\right],\left[\begin{array}{cccc}
0 & 0 & 0 & -\alpha \beta \\
0 & 0 & -\beta & 0 \\
0 & \alpha & 0 & 0 \\
1 & 0 & 0 & 0
\end{array}\right]\right\} .
$$

- Theorem (Teorem) 6: (De Moivre's formula) Let matrix

$$
A=\left[\begin{array}{cccc}
\cos \theta & -\alpha u_{1} \sin \theta & -\beta u_{2} \sin \theta & -\alpha \beta u_{3} \sin \theta \\
u_{1} \sin \theta & \cos \theta & -\beta u_{3} \sin \theta & \beta u_{2} \sin \theta \\
u_{2} \sin \theta & \alpha u_{3} \sin \theta & \cos \theta & -\alpha u_{1} \sin \theta \\
u_{3} \sin \theta & -u_{2} \sin \theta & u_{1} \sin \theta & \cos \theta
\end{array}\right],
$$

correspond to generalized quaternion $q$. The $n$-th power of the matrix $A$ reads as

$$
A^{n}=\left[\begin{array}{cccc}
\cos n \theta & -\alpha u_{1} \sin n \theta & -\beta u_{2} \sin n \theta & -\alpha \beta u_{3} \sin n \theta \\
u_{1} \sin n \theta & \cos n \theta & -\beta u_{3} \sin n \theta & \beta u_{2} \sin n \theta \\
u_{2} \sin n \theta & \alpha u_{3} \sin n \theta & \cos n \theta & -\alpha u_{1} \sin n \theta \\
u_{3} \sin n \theta & -u_{2} \sin n \theta & u_{1} \sin n \theta & \cos n \theta
\end{array}\right] .
$$

Proof: The proof is easily followed by induction on $n$.

- Example (Örnek) 1: Let $q=\frac{1}{2}+\frac{1}{2}\left(\frac{1}{\sqrt{\alpha}}, \frac{1}{\sqrt{\beta}}, \frac{1}{\sqrt{\alpha \beta}}\right)=\cos \frac{\pi}{3}+\vec{u} \sin \frac{\pi}{3}$ be a unit generalized quarternion. The matrix corresponding to this quaternion is

$$
A=\left[\begin{array}{cccc}
\frac{1}{2} & -\frac{\sqrt{\alpha}}{2} & -\frac{\sqrt{\beta}}{2} & -\frac{\sqrt{\alpha \beta}}{2} \\
\frac{1}{2 \sqrt{\alpha}} & \frac{1}{2} & -\frac{\sqrt{\beta}}{2 \sqrt{\alpha}} & \frac{\sqrt{\beta}}{2} \\
\frac{1}{2 \sqrt{\beta}} & \frac{\sqrt{\alpha}}{2 \sqrt{\beta}} & \frac{1}{2} & -\frac{\sqrt{\alpha}}{2} \\
\frac{1}{2 \sqrt{\alpha \beta}} & -\frac{1}{2 \sqrt{\beta}} & \frac{1}{2 \sqrt{\alpha}} & \frac{1}{2}
\end{array}\right],
$$


every power of this matrix with the aid of Theorem 6 is found to be expressible similarly, for example, 28-this

$$
A^{28}=\left[\begin{array}{cccc}
-\frac{1}{2} & \frac{\sqrt{\alpha}}{2} & \frac{\sqrt{\beta}}{2} & \frac{\sqrt{\alpha \beta}}{2} \\
-\frac{1}{2 \sqrt{\alpha}} & -\frac{1}{2} & \frac{\sqrt{\beta}}{2 \sqrt{\alpha}} & -\frac{\sqrt{\beta}}{2} \\
-\frac{1}{2 \sqrt{\beta}} & -\frac{\sqrt{\alpha}}{2 \sqrt{\beta}} & -\frac{1}{2} & \frac{\sqrt{\alpha}}{2} \\
-\frac{1}{2 \sqrt{\alpha \beta}} & \frac{1}{2 \sqrt{\beta}} & -\frac{1}{2 \sqrt{\alpha}} & -\frac{1}{2}
\end{array}\right] .
$$

- Definition (Tanım) 6: (Euler's formula) Let

$$
A=\left[\begin{array}{cccc}
0 & -\alpha u_{1} & -\beta u_{2} & -\alpha \beta u_{3} \\
u_{1} & 0 & -\beta u_{3} & \beta u_{2} \\
u_{2} & \alpha u_{3} & 0 & -\alpha u_{1} \\
u_{3} & -u_{2} & u_{1} & 0
\end{array}\right],
$$

be a real matrix. One immediately finds $A^{2}=-I_{4}$. We have a natural generalization of Euler's formula for matrix $A$;

$$
\begin{aligned}
e^{A \theta} & =I_{4}+A \theta+\frac{(A \theta)^{2}}{2 !}+\frac{(A \theta)^{3}}{3 !}+\frac{(A \theta)^{4}}{4 !}+\ldots \\
& =I_{4}\left(1-\frac{\theta^{2}}{2 !}+\frac{\theta 4}{4 !}-\ldots\right)+A\left(\theta-\frac{\theta^{3}}{3 !}+\frac{\theta^{5}}{5 !}-\ldots\right) \\
& =I_{4} \cos \theta+A \cdot \sin \theta \\
& =I_{4} \cos \theta+\left[\begin{array}{cccc}
0 & -\alpha u_{1} & -\beta u_{2} & -\alpha \beta u_{3} \\
u_{1} & 0 & -\beta u_{3} & \beta u_{2} \\
u_{2} & \alpha u_{3} & 0 & -\alpha u_{1} \\
u_{3} & -u_{2} & u_{1} & 0
\end{array}\right] \cdot \sin \theta \\
& =\left[\begin{array}{cccc}
\cos \theta & -\alpha u_{1} \sin \theta & -\beta u_{2} \sin \theta & -\alpha \beta u_{3} \sin \theta \\
u_{1} \sin \theta & \cos \theta & -\beta u_{3} \sin \theta & \beta u_{2} \sin \theta \\
u_{2} \sin \theta & -\alpha u_{3} \sin \theta & \cos \theta & -\alpha u_{1} \sin \theta \\
u_{3} \sin \theta & -u_{2} \sin \theta & u_{1} \sin \theta & \cos \theta
\end{array}\right] .
\end{aligned}
$$

Let $q=\cos \theta+\vec{u} \sin \theta$ be a unit generalized quaternion. The matrix associated with this quaternion $q$ is of the form (3). In a more general case, we substitute the matrix (3) by

$$
A=\left[\begin{array}{cccc}
\cos (\theta+2 k \pi) & -\alpha u_{1} \sin (\theta+2 k \pi) & -\beta u_{2} \sin (\theta+2 k \pi) & -\alpha \beta u_{3} \sin (\theta+2 k \pi) \\
u_{1} \sin (\theta+2 k \pi) & \cos (\theta+2 k \pi) & -\beta u_{3} \sin (\theta+2 k \pi) & \beta u_{2} \sin (\theta+2 k \pi) \\
u_{2} \sin (\theta+2 k \pi) & -\alpha u_{3} \sin (\theta+2 k \pi) & \cos (\theta+2 k \pi) & -\alpha u_{1} \sin (\theta+2 k \pi) \\
u_{3} \sin (\theta+2 k \pi) & -u_{2} \sin (\theta+2 k \pi) & u_{1} \sin (\theta+2 k \pi) & \cos (\theta+2 k \pi)
\end{array}\right],
$$

where $k \in \mathrm{Z}$. The equation $x^{n}=A$ has $n$ roots, and they are as follows 


$$
A_{k}^{\frac{1}{n}}=\left[\begin{array}{cccc}
\cos \left(\frac{\theta+2 k \pi}{n}\right) & -\alpha u_{1} \sin \left(\frac{\theta+2 k \pi}{n}\right) & -\beta u_{2} \sin \left(\frac{\theta+2 k \pi}{n}\right) & -\alpha \beta u_{3} \sin \left(\frac{\theta+2 k \pi}{n}\right) \\
u_{1} \sin \left(\frac{\theta+2 k \pi}{n}\right) & \cos \left(\frac{\theta+2 k \pi}{n}\right) & -\beta u_{3} \sin \left(\frac{\theta+2 k \pi}{n}\right) & \beta u_{2} \sin \left(\frac{\theta+2 k \pi}{n}\right) \\
u_{2} \sin \left(\frac{\theta+2 k \pi}{n}\right) & -\alpha u_{3} \sin \left(\frac{\theta+2 k \pi}{n}\right) & \cos \left(\frac{\theta+2 k \pi}{n}\right) & -\alpha u_{1} \sin \left(\frac{\theta+2 k \pi}{n}\right) \\
u_{3} \sin \left(\frac{\theta+2 k \pi}{n}\right) & -u_{2} \sin \left(\frac{\theta+2 k \pi}{n}\right) & u_{1} \sin \left(\frac{\theta+2 k \pi}{n}\right) & \cos \left(\frac{\theta+2 k \pi}{n}\right)
\end{array}\right] .
$$

For $k=0$, the first root is

$$
A_{0}^{\frac{1}{n}}=\left[\begin{array}{cccc}
\cos \left(\frac{\theta}{n}\right) & -\alpha u_{1} \sin \left(\frac{\theta}{n}\right) & -\beta u_{2} \sin \left(\frac{\theta}{n}\right) & -\alpha \beta u_{3} \sin \left(\frac{\theta}{n}\right) \\
u_{1} \sin \left(\frac{\theta}{n}\right) & \cos \left(\frac{\theta}{n}\right) & -\beta u_{3} \sin \left(\frac{\theta}{n}\right) & \beta u_{2} \sin \left(\frac{\theta}{n}\right) \\
u_{2} \sin \left(\frac{\theta}{n}\right) & -\alpha u_{3} \sin \left(\frac{\theta}{n}\right) & \cos \left(\frac{\theta}{n}\right) & -\alpha u_{1} \sin \left(\frac{\theta}{n}\right) \\
u_{3} \sin \left(\frac{\theta}{n}\right) & -u_{2} \sin \left(\frac{\theta}{n}\right) & u_{1} \sin \left(\frac{\theta}{n}\right) & \cos \left(\frac{\theta}{n}\right)
\end{array}\right],
$$

and for $k=1$, the second root is

$$
A_{1}^{\frac{1}{n}}=\left[\begin{array}{cccc}
\cos \left(\frac{\theta+2 \pi}{n}\right) & -\alpha u_{1} \sin \left(\frac{\theta+2 \pi}{n}\right) & -\beta u_{2} \sin \left(\frac{\theta+2 \pi}{n}\right) & -\alpha \beta u_{3} \sin \left(\frac{\theta+2 \pi}{n}\right) \\
u_{1} \sin \left(\frac{\theta+2 \pi}{n}\right) & \cos \left(\frac{\theta+2 \pi}{n}\right) & -\beta u_{3} \sin \left(\frac{\theta+2 \pi}{n}\right) & \beta u_{2} \sin \left(\frac{\theta+2 \pi}{n}\right) \\
u_{2} \sin \left(\frac{\theta+2 \pi}{n}\right) & -\alpha u_{3} \sin \left(\frac{\theta+2 \pi}{n}\right) & \cos \left(\frac{\theta+2 \pi}{n}\right) & -\alpha u_{1} \sin \left(\frac{\theta+2 \pi}{n}\right) \\
u_{3} \sin \left(\frac{\theta+2 \pi}{n}\right) & -u_{2} \sin \left(\frac{\theta+2 \pi}{n}\right) & u_{1} \sin \left(\frac{\theta+2 \pi}{n}\right) & \cos \left(\frac{\theta+2 \pi}{n}\right)
\end{array}\right] .
$$

Similarly, for $k=n-1$, we obtain the $n$-th root.

Some relations between the powers of matrices associated with a generalized quaternion is sketched in the following Theorem.

- Theorem (Teorem) 7: Let $q$ be a unit generalized quaternion with the polar form $q=\cos \theta+\vec{u} \sin \theta$. And let $m=\frac{2 \pi}{\theta} \in \mathrm{Z}^{+}-\{1\}$ and the matrix $A$ correspond to $q$. Then $n \equiv p(\bmod m)$ is true if and only if $A^{n}=A^{p}$.

Proof: The proof follows easily from the induction on $n$.

- Example (Örnek) 2:

Let $\quad q=-\frac{1}{2}+\frac{1}{2}\left(\frac{1}{\sqrt{\alpha}}, \frac{1}{\sqrt{\beta}}, \frac{1}{\sqrt{\alpha \beta}}\right)=\cos \frac{2 \pi}{3}+\frac{1}{\sqrt{3}}\left(\frac{1}{\sqrt{\alpha}}, \frac{1}{\sqrt{\beta}}, \frac{1}{\sqrt{\alpha \beta}}\right) \cdot \sin \frac{2 \pi}{3} \quad$ be $\quad$ a unit generalized quarternion. The matrix corresponding to this quaternion is 


$$
A=\left[\begin{array}{cccc}
-\frac{1}{2} & -\frac{\sqrt{\alpha}}{2} & -\frac{\sqrt{\beta}}{2} & -\frac{\sqrt{\alpha \beta}}{2} \\
\frac{1}{2 \sqrt{\alpha}} & -\frac{1}{2} & -\frac{\sqrt{\beta}}{2 \sqrt{\alpha}} & \frac{\sqrt{\beta}}{2} \\
\frac{1}{2 \sqrt{\beta}} & \frac{\sqrt{\alpha}}{2 \sqrt{\beta}} & -\frac{1}{2} & -\frac{\sqrt{\alpha}}{2} \\
\frac{1}{2 \sqrt{\alpha \beta}} & -\frac{1}{2 \sqrt{\beta}} & \frac{1}{2 \sqrt{\alpha}} & -\frac{1}{2}
\end{array}\right]
$$

The square roots of the matrix $A$ can be calculated as follows:

$$
A_{k}^{\frac{1}{2}}=\left[\begin{array}{cccc}
\cos \left(\frac{2 k \pi+2 \pi / 3}{2}\right) & -\alpha u_{1} \sin \left(\frac{2 k \pi+2 \pi / 3}{2}\right) & -\beta u_{2} \sin \left(\frac{2 k \pi+2 \pi / 3}{2}\right) & -\alpha \beta u_{3} \sin \left(\frac{2 k \pi+2 \pi / 3}{2}\right) \\
u_{1} \sin \left(\frac{2 k \pi+2 \pi / 3}{2}\right) & \cos \left(\frac{2 k \pi+2 \pi / 3}{2}\right) & -\beta u_{1} \sin \left(\frac{2 k \pi+2 \pi / 3}{2}\right) & \beta u_{2} \sin \left(\frac{2 k \pi+2 \pi / 3}{2}\right) \\
u_{2} \sin \left(\frac{2 k \pi+2 \pi / 3}{2}\right) & \alpha u_{3} \sin \left(\frac{2 k \pi+2 \pi / 3}{2}\right) & \cos \left(\frac{2 k \pi+2 \pi / 3}{2}\right) & -\alpha u_{1} \sin \left(\frac{2 k \pi+2 \pi / 3}{2}\right) \\
u_{3} \sin \left(\frac{2 k \pi+2 \pi / 3}{2}\right) & -u_{2} \sin \left(\frac{2 k \pi+2 \pi / 3}{2}\right) & u_{1} \sin \left(\frac{2 k \pi+2 \pi / 3}{2}\right) & \cos \left(\frac{2 k \pi+2 \pi / 3}{2}\right)
\end{array}\right]
$$

The first root for $k=0$ is

$$
A_{0}^{\frac{1}{2}}=\left[\begin{array}{cccc}
\frac{1}{2} & -\frac{\sqrt{\alpha}}{2} & -\frac{\sqrt{\beta}}{2} & -\frac{\sqrt{\alpha \beta}}{2} \\
\frac{1}{2 \sqrt{\alpha}} & \frac{1}{2} & -\frac{\sqrt{\beta}}{2 \sqrt{\alpha}} & \frac{\sqrt{\beta}}{2} \\
\frac{1}{2 \sqrt{\beta}} & \frac{\sqrt{\alpha}}{2 \sqrt{\beta}} & \frac{1}{2} & -\frac{\sqrt{\alpha}}{2} \\
\frac{1}{2 \sqrt{\alpha \beta}} & -\frac{1}{2 \sqrt{\beta}} & \frac{1}{2 \sqrt{\alpha}} & \frac{1}{2}
\end{array}\right],
$$

and the second one for $k=1$ is

$$
A_{1}^{\frac{1}{2}}=\left[\begin{array}{cccc}
-\frac{1}{2} & \frac{\sqrt{\alpha}}{2} & \frac{\sqrt{\beta}}{2} & \frac{\sqrt{\alpha \beta}}{2} \\
-\frac{1}{2 \sqrt{\alpha}} & -\frac{1}{2} & \frac{\sqrt{\beta}}{2 \sqrt{\alpha}} & -\frac{\sqrt{\beta}}{2} \\
-\frac{1}{2 \sqrt{\beta}} & -\frac{\sqrt{\alpha}}{2 \sqrt{\beta}} & -\frac{1}{2} & \frac{\sqrt{\alpha}}{2} \\
-\frac{1}{2 \sqrt{\alpha \beta}} & \frac{1}{2 \sqrt{\beta}} & -\frac{1}{2 \sqrt{\alpha}} & -\frac{1}{2}
\end{array}\right] \text {. }
$$

Also, it is easy to see that $A_{0}^{\frac{1}{2}}+A_{1}^{\frac{1}{2}}=0$.

From the Theorem 7, with $m=\frac{2 \pi}{2 \pi / 3}=3$, we get

$$
\begin{aligned}
& A=A^{4}=A^{7}=A^{10}=\ldots \\
& A^{2}=A^{5}=A^{8}=A^{11}=\ldots \\
& A^{3}=A^{6}=A^{9}=A^{12}=\ldots=I_{4} .
\end{aligned}
$$




\section{Jafari, $M$. \\ NWSA-Physical Sciences, 3A0067, 10, (1), 1-13.}

- Case (Durum) 2: Let $\alpha$ be a positive number and $\beta$ be a negative number. In this case, the Theorems 6 holds.

In following Theorem, we show how unit quaternions can be used to described the rotation in 4-space $\mathrm{E}_{\alpha \beta}^{4}$.

- Theorem (Teorem) 8: Let $q$ be a unit generalized quaternion. Matrices generated by operators $\stackrel{+}{H}$ and $\bar{H}$ are quasi-orthogonal matrices, i.e.

i)

$$
\begin{aligned}
& {[\stackrel{+}{H}(q)]^{T} \varepsilon \stackrel{+}{H}(q)=\varepsilon,} \\
& {[\vec{H}(q)]^{T} \varepsilon \stackrel{-}{H}(q)=\varepsilon, \varepsilon=\left[\begin{array}{cccc}
1 & 0 & 0 & 0 \\
0 & \alpha & 0 & 0 \\
0 & 0 & \beta & 0 \\
0 & 0 & 0 & \alpha \beta
\end{array}\right] .}
\end{aligned}
$$

- Corollary (Sonuç) 1: Let $q=\cos \theta+\vec{u} \sin \theta$ be a unit generalized quaternion. Then the generalized Hamilton operators $\hat{h}_{q}^{+}$and $\bar{h}_{q}$ represent rotations of $x$ in $\mathrm{E}_{\alpha \beta}^{4}$.

The angle of rotation (using $\stackrel{+}{h}_{q}$ ) is easily determined. This is the angle $\omega$ between $x$ and $q x$ :

$$
\begin{aligned}
\cos \omega & =\frac{S(x(\overline{q x}))}{\sqrt{N_{x}} \sqrt{N_{q x}}} \\
& =\frac{S(x(\bar{x} \bar{q}))}{N_{x} \sqrt{N_{q}}}=\frac{S(q)}{\sqrt{N_{q}}}=S(q)=\cos \theta .
\end{aligned}
$$

Therefore that the angle of rotation $\omega$ is the angle of $q$.

- Example (Örnek) 3: Let $q=\frac{1}{\sqrt{2}}+\frac{1}{2}\left(\frac{1}{\sqrt{\alpha}}, \frac{1}{\sqrt{\beta}}, 0\right)$ be a unit generalized quaternion and $\alpha, \beta>0$. The matrix corresponding to this quaternion is

$$
A=\frac{1}{2}\left[\begin{array}{cccc}
\sqrt{2} & -\sqrt{\alpha} & -\sqrt{\beta} & 0 \\
\frac{1}{\sqrt{\alpha}} & \sqrt{2} & 0 & \sqrt{\beta} \\
\frac{1}{\sqrt{\beta}} & 0 & \sqrt{2} & -\sqrt{\alpha} \\
0 & -\frac{1}{\sqrt{\beta}} & \frac{1}{\sqrt{\alpha}} & \sqrt{2}
\end{array}\right] .
$$

$A$ is a quasi-orthogonal matrix and therefore it represents a rotation in 4 -space $\mathrm{E}_{\alpha \beta}^{4}$. 


\section{Jafari, $M$. NWSA-Physical Sciences, 3A0067, 10, (1), 1-13.}

In following, we show how matrices corresponding to generalized quaternion can be used to described the homothetic motion 4-space $\mathrm{E}_{\alpha \beta}^{4}$. Let us consider the following curve:

$$
a: I \subset \mathrm{R} \rightarrow \mathrm{E}_{\alpha \beta}^{4}
$$

defined by $a(t)=\left(a_{0}(t), a_{1}(t), a_{2}(t), a_{3}(t)\right)$ for every $t \in I$.

We suppose that the unit velocity curve $a(t)$ is differentiable regular curve of order $r$ The operator B called the Hamiltonian operator, corresponding to $a(t)$ is defined by the following matrix;

$$
B=\stackrel{+}{H}[a(t)]=\left[\begin{array}{cccc}
a_{0}(t) & -\alpha a_{1}(t) & -\beta a_{2}(t) & -\alpha \beta a_{3}(t) \\
a_{1}(t) & a_{0}(t) & -\beta a_{3}(t) & \beta a_{2}(t) \\
a_{2}(t) & \alpha a_{3}(t) & a_{0}(t) & -\alpha a_{1}(t) \\
a_{3}(t) & -a_{2}(t) & a_{1}(t) & a_{0}(t)
\end{array}\right] .
$$

- Definition (Tanım) 9. The 1-parameter Hamilton motions of a body in $\mathrm{E}_{\alpha \beta}^{4}$ are generated by transformation

$$
\left[\begin{array}{l}
Y \\
1
\end{array}\right]=\left[\begin{array}{ll}
B & C \\
0 & 1
\end{array}\right]\left[\begin{array}{l}
X \\
1
\end{array}\right]
$$

or equivalently

$$
Y=B X+C
$$

Here $B=\stackrel{+}{H}[a(t)]$ and $Y, X$ and $C$ are $n \times 1$ real matrices. $Y$ and $X$ correspond to the position vectors of the same point $P$.

- Theorem (Teorem) 9: The Hamilton motion determined by equation (4) is a homothetic motion in $\mathrm{E}_{\alpha \beta}^{4}$.

Proof: We suppose that length of $a(t)$ is not zero, so the matrix $B$ can be represented as

where $h: I \subset \mathrm{R} \rightarrow \mathrm{R}$,

$$
B=h\left[\begin{array}{cccc}
\frac{a_{0}(t)}{h} & -\frac{\alpha a_{1}(t)}{h} & -\frac{\beta a_{2}(t)}{h} & -\frac{\alpha \beta a_{3}(t)}{h} \\
\frac{a_{1}(t)}{h} & \frac{a_{0}(t)}{h} & -\frac{\beta a_{3}(t)}{h} & \frac{\beta a_{2}(t)}{h} \\
\frac{a_{2}(t)}{h} & \frac{\alpha a_{3}(t)}{h} & \frac{a_{0}(t)}{h} & -\frac{\alpha a_{1}(t)}{h} \\
\frac{a_{3}(t)}{h} & \frac{-a_{2}(t)}{h} & \frac{a_{1}(t)}{h} & \frac{a_{0}(t)}{h}
\end{array}\right]
$$

$$
t \rightarrow h(t)=\|\alpha(t)\|=\sqrt{a_{0}^{2}(t)+\alpha a_{1}^{2}(t)+\beta a_{2}^{2}(t)+\alpha \beta a_{3}^{2}(t) .} .
$$

So, we find $A^{T} \varepsilon A=\varepsilon$ and $\operatorname{det} A=1$, thus $\mathrm{B}$ is a homothetic matrix and equation (4) determines a homothetic motion. For detailed information about the homothetic motions; we refer the reader to [2]. 


\section{CONCLUSION (SONUÇ)}

With the aid of the Hamilton operators, generalized quaternions have been expressed in terms of $4 \times 4$ matrices. In this paper, algebraic properties and geometric applications of these matrices in generalized 4-space $\mathrm{E}_{\alpha \beta}^{4}$ are studied. Also, it is shown that the set of these matrices with the group operation of matrix multiplication is a Lie group of 6-dimension and its Lie algebra is found.

\section{REFERENCES (KAYNAKLAR)}

1. Jafari, M. and Yaylı, Y., (2013). Rotation in four dimensions via generalized Hamilton operators, Kuwait journal of science, Volume:40, Number:1, pp:45-56.

2. Jafari, M., and Yaylı, Y., (2010). Homothetic motions at $\mathrm{E}_{\alpha \beta}^{4}$. International Journal Contemporary of Mathematics Sciences. Volume:5, Number:47, pp:2319-2326.

3. Mamagani, A.B. and Jafari, M., (2013). Some notes on matrix of generalized quaternion, Volume:7, Number:14, pp: 1086-1093.

4. Meinrenken, E., (2010). Lie groups and Lie algebras, Lecture Notes, University of Toronto.

5. Pottman, H. and Wallner, J., (2000). Computational line geometry. Springer-Verlag, New York.

6. Unger, T. and Markin, N., (2008). Quadratic forms and space-time block codes from generalized quaternion and biquaternion algebras. IEEE transactions on information theory, Volume:57, Number:9, pp: 6148-6156.

7. Ward, J.P., (1997). Quaternions and Cayley numbers algebra and applications, Kluwer Academic Publishers, London. 